\title{
The anal exam and SCI
}

\author{
Marcalee Alexander $^{1}$
}

Received: 27 September 2017 / Accepted: 18 October 2017

(C) International Spinal Cord Society 2017

I am excited to bring to you the first Spinal Cord Series and Cases Pulse and Point-Counter Point articles. In the journal aims and scope we state, "The journal strongly supports the use of the International Data Sets, the International Standards to Document Remaining Autonomic Function after Spinal Cord Injury and the International Standards for the Neurologic Classification of SCI in submitted publications and encourages papers that highlight the educational aspects of these documents." Hence, our first Pulse article highlights potential variability in performance of the anal examination, and our first Point Counterpoint articles highlight the importance of the anal examination and the associated bulbocavernosus reflex. As can be seen, although the ISNCSCI has been established for many years, there are still questions about the exam and its meaning that need to be asked. Specifically, the consistency of the exam, the issue of motor recovery vs. autonomic recovery, and what components are needed in the exam to predict recovery of different functions are addressed. I encourage you to read these papers and hope they spark discussion and further related case reports and pilot studies. I also hope they help start a dialog about cure research and whether we are effectively assessing all outcomes, especially with respect to autonomic recovery.

Although promoting recovery is an important topic, it is only the tip of the iceberg. As providers, we need to be realistic and responsive to all aspects of the complicated lives of persons with SCIs. Living with a SCI is undoubtedly more difficult than living as able-bodied person. Therefore, one of the goals of SCSC is to help providers understand the day-to-day lives of persons with SCIs so we can be more effective, understanding, and knowledgeable.

Ten years ago at the ISCOS meeting in Rekjavik [1], I spoke about the need for a greater intersection between

Marcalee Alexander

spinalcordmd@live.com

1 University of Alabama School of Medicine, Birmingham, Birmingham Veterans Affairs Medical Center, Birmingham AL, USA what we do for our patients as providers and researchers and the issues associated with climate change. With recent catastrophic weather and climate-based news, the difficulties with living with SCI have become more germane not only for persons in developing countries but also in the developed world. In light of this, in conjunction with the ISCOS disaster committee, SCSC will host a Pulse Survey about weather and natural or war-related disaster issues and what we need to do as clinicians to gain knowledge to deal with them. I would also like to receive related submissions to SCSC about how these concerns have impacted the lives of persons with SCIs. I want to hear about the problems that have occurred and how you have worked to help your patients. Moreover, I would like to highlight the mechanisms that have helped you care for patients in times of disaster, what the difficulties have been, and what we need to study and address to prepare for the future.

In addition to living with ongoing changes in our lives for persons with SCIs, we must also take care of ourselves. Now more than ever the adage "Put on your own life mask first" is meaningful. In speaking with colleagues in all fields and all countries, the issues of excessive need for documentation, insurance issues, political uncertainty, excessive work hours, and lack of resources seem to be bearing down on our energy levels. Therefore, we have put together a PULSE survey with regards to meaning and burnout for SCI providers. The link follows and if you work in SCI please complete the survey if you have not already https:// www.surveymonkey.com/r/TGTQHNK.

I hope you enjoy these new features in Spinal Cord Series and Cases and will participate in our Pulse Surveys. Remember this is your journal, the place where the seeds of knowledge related to SCI are planted and ideas blossom! As always, feel free to contact me with any ideas or suggestions.

\section{References}

1. Alexander MS. Autonomic function and SCI: are we at a crossroads? Spinal Cord. 2008;46:402-5 